Proyecciones Journal of Mathematics

Vol. 40, No 5, pp. 1197-1209, October 2021.

Universidad Católica del Norte

Antofagasta - Chile

\title{
A new generalized refinements of Young's inequality
}

Mohamed Amine Ighachane

University Cadi Ayyad, Morocco

and

Mohamed Akkouchi

University Cadi Ayyad, Morocco

Received : August 2020. Accepted : March 2021

\begin{abstract}
In this paper, we show a new generalized refinement of Young's inequality. As applications we give some new generalized refinements of Young's type inequalities for the determinants, traces and norms of positive definite matrices.
\end{abstract}

Subjclass [2010]: 15A45; 15A60; 26D0\%.

Keywords: AM-GM inequality; Young inequality; Determinants; Trace; Norms 


\section{Introduction}

The arithmetic-geometric mean (AM-GM) inequality states as follows:

Theorem 1.1. Let $n$ be a positive integer. For $k=1,2, \ldots, n$, let $x_{k}>0$, and let $\nu_{k} \geq 0$ satisfy $\sum_{k=1}^{n} \nu_{k}=1$. Then, we have

$$
\prod_{k=1}^{n} x_{k}^{\nu_{k}} \leq \sum_{k=1}^{n} \nu_{k} x_{k}
$$

The special case of the weighted AM-GM inequality $(n=2)$ is the wellknown Young's inequality, for positive real numbers $a, b$ and $0 \leq \nu \leq 1$, we have

$$
a^{\nu} b^{1-\nu} \leq \nu a+(1-\nu) b .
$$

The first refinements of Young inequality is the squared version proved in [7] as follows

$$
\left(a^{\nu} b^{1-\nu}\right)^{2}+r_{0}^{2}(a-b)^{2} \leq(\nu a+(1-\nu) b)^{2},
$$

where $r_{0}=\min \{\nu, 1-\nu\}$.

Recently, Kittaneh and Manasrah [12] refined Young's inequality so that

$$
a^{\nu} b^{1-\nu}+r_{0}(\sqrt{a}-\sqrt{b})^{2} \leq \nu a+(1-\nu) b,
$$

where $r_{0}=\min \{\nu, 1-\nu\}$.

Later, J. Zhao and J. Wu [13], obtained the following refinement of inequality (1.2) as follows

$$
\begin{gathered}
{ }^{\nu} b^{1-\nu}+r_{0}(\sqrt{a}-\sqrt{b})^{2}+r_{1}\left((\sqrt[4]{a b}-\sqrt{b})^{2} \chi_{\left(0, \frac{1}{2}\right]}(\nu)+(\sqrt[4]{a b}-\sqrt{a})^{2} \chi_{\left(\frac{1}{2}, 1\right]}(\nu)\right) \\
\leq \nu a+(1-\nu) b
\end{gathered}
$$

where $r_{0}=\min \{\nu, 1-\nu\}$ and $r_{1}=\min \left\{2 r_{0}, 1-2 r_{0}\right\}$ and $\chi_{I}$ the characteristic function defined by

$$
\chi_{I}(x)= \begin{cases}1 & \text { if } x \in I \\ 0 & \text { if } x \notin I .\end{cases}
$$


S. Furuichi [5] was refined (1.1) as follows:

$$
\prod_{k=1}^{n} x_{k}^{\nu_{k}}+r\left(\sum_{k=1}^{n} x_{k}-n \sqrt[n]{\prod_{k=1}^{n} x_{k}}\right) \leq \sum_{k=1}^{n} \nu_{k} x_{k},
$$

where $r=\min \left\{\nu_{k}: k=1, \ldots, n\right\}$. This inequality generalizes the inequality (1.4).

For a generalized refinement of the weighted arithmetic-geometric mean inequality see [9].

Manasrah and Kittaneh [1] gave generalized refinements of the inequalities (1.3) and (1.4). as follows

Theorem 1.2. If $a, b>0$ and $0 \leq \nu \leq 1$, then for $m=1,2,3, \ldots$ we have

$$
\left(a^{\nu} b^{1-\nu}\right)^{m}+r_{0}^{m}\left(a^{\frac{m}{2}}-b^{\frac{m}{2}}\right)^{2} \leq(\nu a+(1-\nu) b)^{m},
$$

where $m=1,2,3, \ldots$, and $r_{0}=\min \{\nu, 1-\nu\}$.

Recently, Manasrah and Kittaneh [2] gave further generalizations and refinements of (1.3) and (1.4) as follows

Theorem 1.3. If $a, b>0$ and $0 \leq \nu \leq 1$, then for $m=1,2,3, \ldots$, we have

$r_{0}^{m}\left(a^{\frac{m}{2}}-b^{\frac{m}{2}}\right)^{2} \leq r_{0}^{m}\left((a+b)^{m}-2^{m}(a b)^{\frac{m}{2}}\right) \leq(\nu a+(1-\nu) b)^{m}-\left(a^{\nu} b^{1-\nu}\right)^{m}$,

where $r_{0}=\min \{\nu, 1-\nu\}$.

For more other general refinement of inequalities (1.3) and (1.4) see [10].

One of the aims of this paper is to refine the second inequality of Theorem 1.3 by adding the positive quantity :

$$
\begin{gathered}
r_{m}\left[\left(b\left(\frac{b^{m}-a^{m}}{b-a}\right)+(a b)^{\frac{m}{2}}-(m+1)\left(a^{m} b^{m+2}\right)^{\frac{m}{2(m+1)}}\right) \chi_{\left(0, \frac{1}{2}\right]}(\nu)+\right. \\
\left.\left.\left(a\left(\frac{b^{m}-a^{m}}{b-a}\right)+(a b)^{\frac{m}{2}}-(m+1)\left(a^{m+2} b^{m}\right)^{\frac{m}{2(m+1)}}\right) \chi_{\left(\frac{1}{2}, 1\right)}(\nu)\right)\right],
\end{gathered}
$$


in the first part. Where $r_{0}=\min \{\nu, 1-\nu\}, r_{m}=\min \left\{2^{m} r_{0}^{m} ;\left(\begin{array}{c}m \\ k\end{array}\right)\left(r_{0}^{k}(1-\right.\right.$ $\left.\left.\left.r_{0}\right)^{m-k}-r_{0}^{m}\right), 0 \leq k \leq m-1\right\}$ and $\chi_{I}(\nu)$ the characteristic function. As applications we give some new generalized refinements of Young's type inequalities for the determinants, traces and norms of positive definite matrices.

\section{Generalized refinements of Young's inequality}

In this section, we prove the main result of this paper. To do this, we need the following lemma.

Lemma 2.1. Let $m$ be a positive integer, and let $\nu$ a positive number such that $0 \leq \nu \leq 1$. Then we have

$$
\sum_{k=1}^{m}\left(\begin{array}{c}
m \\
k
\end{array}\right) k \nu^{k}(1-\nu)^{m-k}=m \nu
$$

and

$$
\begin{aligned}
& \sum_{k=0}^{m-1}\left(\begin{array}{c}
m \\
k
\end{array}\right)(m-k) \nu^{k}(1-\nu)^{m-k}=m(1-\nu), \\
& \sum_{k=1}^{m-1}\left(\begin{array}{c}
m \\
k
\end{array}\right) k=\sum_{k=0}^{m-1}\left(\begin{array}{c}
m \\
k
\end{array}\right)(m-k)=m 2^{m-1},
\end{aligned}
$$

where $\left(\begin{array}{c}m \\ k\end{array}\right)$ is the binomial coefficient.

For a proof of Lemma 2.1, one can see [3].

The main result to be proved in this paper is:

Theorem 2.1. Let $a$ and $b$ be two positive numbers and $0 \leq \nu \leq 1$. Then for $m=1,2,3, \ldots$, we have

$$
\begin{aligned}
\left(a^{\nu} b^{1-\nu}\right)^{m}+ & r_{0}^{m}\left((a+b)^{m}-2^{m}(a b)^{\frac{m}{2}}\right) \\
+ & r_{m}\left[\left(b\left(\frac{b^{m}-a^{m}}{b-a}\right)+(a b)^{\frac{m}{2}}-(m+1)\left(a^{m} b^{m+2}\right)^{\frac{m}{2(m+1)}}\right) \chi_{\left(0, \frac{1}{2}\right]}(\nu)+\right. \\
& \left.\left.\left(a\left(\frac{b^{m}-a^{m}}{b-a}\right)+(a b)^{\frac{m}{2}}-(m+1)\left(a^{m+2} b^{m}\right)^{\frac{m}{2(m+1)}}\right) \chi_{\left(\frac{1}{2}, 1\right)}(\nu)\right)\right] \\
\leq & (\nu a+(1-\nu) b)^{m},
\end{aligned}
$$


where $r_{0}=\min \{\nu, 1-\nu\}, r_{m}=\min \left\{2^{m} r_{0}^{m} ;\left(\begin{array}{c}m \\ k\end{array}\right) r_{0}^{k}\left(1-r_{0}\right)^{m-k}, 0 \leq k \leq\right.$ $m-1\}$, and $\chi_{I}(\nu)$ the characteristic function.

Proof. Suppose that $0 \leq \nu \leq \frac{1}{2}$. We claim that

$$
\begin{aligned}
\left(a^{\nu} b^{1-\nu}\right)^{m} & +\nu^{m}\left((a+b)^{m}-2^{m}(a b)^{\frac{m}{2}}\right) \\
& +r_{m}\left(b\left(\frac{b^{m}-a^{m}}{b-a}\right)+(a b)^{\frac{m}{2}}-(m+1)\left(a^{m} b^{m+2}\right)^{\frac{m}{2(m+1)}}\right) \\
& \leq(\nu a+(1-\nu) b)^{m} .
\end{aligned}
$$

We have

$$
\begin{aligned}
(\nu a+ & (1-\nu) b)^{m}-\nu^{m}\left((a+b)^{m}-2^{m}(a b)^{\frac{m}{2}}\right) \\
& =\sum_{k=0}^{m}\left(\begin{array}{c}
m \\
k
\end{array}\right) \nu^{k}(1-\nu)^{m-k} a^{k} b^{m-k}-\nu^{m}\left(\sum_{k=0}^{m}\left(\begin{array}{c}
m \\
k
\end{array}\right) a^{k} b^{m-k}-2^{m}(a b)^{\frac{m}{2}}\right) \\
& =\sum_{k=0}^{m-1}\left(\begin{array}{c}
m \\
k
\end{array}\right)\left(\nu^{k}(1-\nu)^{m-k}-\nu^{m}\right) a^{k} b^{m-k}+2^{m} \nu^{m}(a b)^{\frac{m}{2}} \\
& =\sum_{k=0}^{m} \nu_{k} x_{k},
\end{aligned}
$$

where $x_{k}$ is given by: for $0 \leq k \leq m-1$,

$$
x_{k}:=a^{k} b^{m-k}, \quad \text { with } \quad \nu_{k}:=\left(\begin{array}{c}
m \\
k
\end{array}\right)\left(\nu^{k}(1-\nu)^{m-k}-\nu^{m}\right),
$$

and

$$
x_{m}:=(a b)^{\frac{m}{2}}, \quad \text { with } \quad \nu_{m}:=2^{m} \nu^{m} .
$$

We have

1. $x_{k}>0$ for all $k \in\{0,1, \ldots, m\}$,

2. $\nu_{k} \geq 0$ for all $k \in\{0,1, \ldots, m\}$, with $\sum_{k=0}^{m} \nu_{k}=1$.

Hence, by inequality (1.6), 


$$
\begin{aligned}
(\nu a+(1-\nu) b)^{m} & -\nu^{m}\left((a+b)^{m}-2^{m}(a b)^{\frac{m}{2}}\right) \\
& \geq \prod_{k=0}^{m} x_{k}^{\nu_{k}}+r\left(\sum_{k=0}^{m} x_{k}-(m+1) \sqrt[(m+1)]{\prod_{k=0}^{m} x_{k}}\right) \\
= & a^{\alpha(m)} b^{\beta(m)}+r\left(\sum_{k=0}^{m-1} a^{k} b^{m-k}+(a b)^{\frac{m}{2}}-(m+1)\left((a b)^{\frac{m}{2}} \prod_{k=0}^{m-1} a^{k} b^{m-k}\right)^{\frac{1}{m+1}}\right) \\
= & a^{\alpha(m)} b^{\beta(m)}+r\left(b\left(\frac{b^{m}-a^{m}}{b-a}\right)+(a b)^{\frac{m}{2}}-(m+1)\left(a^{m} b^{m+2}\right)^{\frac{m}{2(m+1)}}\right),
\end{aligned}
$$

where $r=\min \left\{\nu_{k}, k=0, \ldots, m\right\}=\min \left\{2^{m} r_{0}^{m} ;\left(\begin{array}{c}m \\ k\end{array}\right)\left(r_{0}^{k}\left(1-r_{0}\right)^{m-k}-\right.\right.$ $\left.\left.r_{0}^{m}\right), 0 \leq k \leq m-1\right\}$,

$$
\begin{aligned}
\alpha(m) & =\sum_{k=1}^{m-1}\left(\begin{array}{c}
m \\
k
\end{array}\right) k\left(\nu^{k}(1-\nu)^{m-k}-\nu^{m}\right)+\frac{m}{2} \cdot 2^{m} \nu^{m} \\
& =\sum_{k=1}^{m}\left(\begin{array}{c}
m \\
k
\end{array}\right) k \nu^{k}(1-\nu)^{m-k}-\nu^{m} \sum_{k=1}^{m}\left(\begin{array}{c}
m \\
k
\end{array}\right) k+2^{m-1} m \nu^{m} \\
& =m \nu, \quad(\text { by Lemma } 2.1)
\end{aligned}
$$

and

$$
\begin{aligned}
\beta(m) & =\sum_{k=0}^{m-1}\left(\begin{array}{c}
m \\
k
\end{array}\right)(m-k)\left(\nu^{k}(1-\nu)^{m-k}-\nu^{m}\right)+\frac{m}{2} \cdot 2^{m} \nu^{m} \\
& =\sum_{k=0}^{m-1}\left(\begin{array}{c}
m \\
k
\end{array}\right)(m-k) \nu^{k}(1-\nu)^{m-k}-\nu^{m} \sum_{k=0}^{m-1}\left(\begin{array}{c}
m \\
k
\end{array}\right)(m-k)+2^{m-1} m \nu^{m} \\
& =m(1-\nu)(\text { by Lemma 2.1by Lemma 2.1by Lemma 2.1by Lemma 2.1). }
\end{aligned}
$$

If $\nu \in\left[\frac{1}{2}, 1\right]$, then $1-\nu \in\left[0, \frac{1}{2}\right]$. So by changing two elements $a, b$ and two weights $\nu, 1-\nu$ in inequality (2.4), the desired inequality is obtained.

Remark 2.1. The above theorem is a generalization of the inequality (1.5) obtained by $J$. Zhao and $J$. Wu in [13], which correspond to the case $m=1$. 


\section{Applications}

In this section, we give some refined Young type inequalities for traces, determinants, and norms of positive definite matrices.

Let $\mathbf{M}_{n}(\mathbf{C})$ be the space of $n \times n$ complex matrices. A matrix $A \in$ $\mathbf{M}_{n}(\mathbf{C})$ is called positive semi-definite, denoted as $A \geq 0$ if $x^{*} A x \geq 0$ for all $x \in \mathbf{C}^{n}$, and it is called positive definite denoted as $A>0$ if $x^{*} A x>0$ for all nonzero $x \in \mathbf{C}^{n}$. The singular values of a matrix $A \in \mathbf{M}_{n}(\mathbf{C})$ are the eigenvalues of the positive semi-definite matrix $|A|=\left(A^{*} A\right)^{1 / 2}$, denoted by $s_{i}(A)$ for $i=1,2,3, \ldots, n$. A norm \|\|$.\|\|$, on $\mathbf{M}_{n}(\mathbf{C})$ is called unitarily invariant if $\||U A V\|\|=\|A\||\|$ for all $A \in \mathbf{M}_{n}(\mathbf{C})$ and all unitary matrices $U, V \in \mathbf{M}_{n}(\mathbf{C})$.

The trace norm is given by $\|A\|_{1}=t r|A|=\sum_{k=1}^{n} s_{k}(A)$, where $t r$ is the usual trace. This norm is unitarily invariant.

A matrix Young's inequality due to Ando [4] asserts that

$$
s_{j}\left(A^{\nu} B^{1-\nu}\right) \leq s_{j}(\nu A+(1-\nu) B),
$$

the above singular value inequality entails the following unitarily invarant norm inequality

$$
||\left|A^{\nu} B^{1-\nu}\right||| \leq|||\nu A+(1-\nu) B||| .
$$

A determinant version of Young's inequalities is also known [6, p. 467]: For positive semi-definite matrices $A, B$ and $0 \leq \nu \leq 1$,

$$
\operatorname{det}\left(A^{\nu} B^{1-\nu}\right) \leq \operatorname{det}(\nu A+(1-\nu) B) .
$$

To prove the result of this section, we need the following two lemmas, the first lemma (see, e.g., [6, p. 482,]) is the Minkowski inequality for determinants. The second lemma [11] is a Heinz-Kato type inequality for unitarily invariant norms.

Lemma 3.1. Let $A, B \in \mathbf{M}_{n}(\mathbf{C})$ be positive definite matrices. Then we have

$$
\operatorname{det}(A+B)^{\frac{1}{n}} \geq \operatorname{det}(A)^{\frac{1}{n}}+\operatorname{det}(B)^{\frac{1}{n}} .
$$

Lemma 3.2. Let $A, B \in \mathbf{M}_{n}(\mathbf{C})$ be positive semi-definite matrices. Then we have

$$
\left\|A ^ { \nu } X B ^ { 1 - \nu } \left|\left\|\leq\left|\left\|A X \left|\left\|\left.\right|^{\nu}|| X B|\||^{1-\nu} .\right.\right.\right.\right.\right.\right.\right.
$$


In particular

$$
\operatorname{tr}\left|A^{\nu} B^{1-\nu}\right| \leq(\operatorname{tr} A)^{\nu}(\operatorname{tr} B)^{1-\nu} .
$$

The first result of this section concerns the determinants of positive definite matrices which can be reads as follows:

Theorem 3.1. Let $A, B \in \mathbf{M}_{n}(\mathbf{C})$ be positive definite matrices and $0 \leq$ $\nu \leq 1$. Then for $m=1,2,3, \ldots$, we have

$$
\begin{gathered}
\left(\operatorname{det}\left(A^{\nu} B^{1-\nu}\right)\right)^{m}+r_{0}^{n m}\left(\left(\operatorname{det}(A)^{\frac{1}{n}}+\operatorname{det}(B)^{\frac{1}{n}}\right)^{n m}-2^{n m}(\operatorname{det}(A B))^{\frac{m}{2}}\right) \\
+r_{n m}\left[\left(\operatorname{det}(B)^{\frac{1}{n}} \frac{\operatorname{det}(B)^{m}-\operatorname{det}(A)^{m}}{\operatorname{det}(B)^{\frac{1}{n}}-\operatorname{det}(A)^{\frac{1}{n}}}+\operatorname{det}(A B)^{\frac{m}{2}}-(n m+1)\left(\operatorname{det}\left(A^{m} B^{m+\frac{2}{n}}\right)\right)^{\frac{n m}{2(n m+1)}}\right)\right. \\
\times \quad \chi_{\left(0, \frac{1}{2}\right]}(\nu) \\
\left.+\left(\operatorname{det}(A)^{\frac{1}{n}} \frac{\operatorname{det}(B)^{m}-\operatorname{det}(A)^{m}}{\operatorname{det}(B)^{\frac{1}{n}}-\operatorname{det}(A)^{\frac{1}{n}}}+\operatorname{det}(A B)^{\frac{m}{2}}-(n m+1)\left(\operatorname{det}\left(A^{m+\frac{2}{n}} B^{m}\right)\right)^{\frac{n m}{2(n m+1)}}\right)\right] \\
\times \chi_{\left(\frac{1}{2}, 1\right]}(\nu) \\
\leq \operatorname{det}(\nu A+(1-\nu) B)^{m},
\end{gathered}
$$

where $r_{0}=\min \{\nu, 1-\nu\}$, and $r_{n m}=\min \left\{2^{n m} r_{0}^{n m} ;\left(\begin{array}{c}n m \\ k\end{array}\right)\left(r_{0}^{k}\left(1-r_{0}\right)^{n m-k}-\right.\right.$ $\left.\left.r_{0}^{n m}\right), 0 \leq k \leq n m-1\right\}$.

Proof. We have

$$
\begin{aligned}
\operatorname{det}(\nu A+(1-\nu) B)^{m} & =\left[\operatorname{det}(\nu A+(1-\nu) B)^{\frac{1}{n}}\right]^{n m} \\
& \geq\left[\operatorname{det}(\nu A)^{\frac{1}{n}}+\operatorname{det}((1-\nu) B)^{\frac{1}{n}}\right]^{n m}(\text { by Lemma 3.1) } \\
& =\left[\nu \operatorname{det}(A)^{\frac{1}{n}}+(1-\nu) \operatorname{det}(B)^{\frac{1}{n}}\right]^{n m} .
\end{aligned}
$$

So,

$$
\begin{aligned}
\operatorname{det}(\nu A+(1-\nu) B)^{m} & \geq\left[\left(\operatorname{det}(A)^{\frac{1}{n}}\right)^{\nu}\left(\operatorname{det}(B)^{\frac{1}{n}}\right)^{1-\nu}\right]^{n m} \\
& +r_{0}^{n m}\left(\left(\operatorname{det}(A)^{\frac{1}{n}}+\operatorname{det}(B)^{\frac{1}{n}}\right)^{n m}-2^{n m}(\operatorname{det}(A B))^{\frac{m}{2}}\right)
\end{aligned}
$$




$$
\begin{aligned}
& +r_{n m}\left[\left(\operatorname{det}(B)^{\frac{1}{n}} \frac{\operatorname{det}(B)^{m}-\operatorname{det}(A)^{m}}{\operatorname{det}(B)^{\frac{1}{n}}-\operatorname{det}(A)^{\frac{1}{n}}}+\operatorname{det}(A B)^{\frac{m}{2}}-(n m+1)\left(\operatorname{det}\left(A^{m} B^{m+\frac{2}{n}}\right)\right)^{\frac{n m}{2(n m+1)}}\right.\right. \\
& \times \chi_{\left(0, \frac{1}{2}\right]}(\nu) \\
& \left.+\left(\operatorname{det}(A)^{\frac{1}{n}} \frac{\operatorname{det}(B)^{m}-\operatorname{det}(A)^{m}}{\operatorname{det}(B)^{\frac{1}{n}}-\operatorname{det}(A)^{\frac{1}{n}}}+\operatorname{det}(A B)^{\frac{m}{2}}-(n m+1)\left(\operatorname{det}\left(A^{m+\frac{2}{n}} B^{m}\right)\right)^{\frac{n m}{2(n m+1)}}\right)\right] \\
& \times \chi_{\left(\frac{1}{2}, 1\right]}(\nu) \\
& \text { (by Theorem 2.1) } \\
& =\left(\operatorname{det}\left(A^{\nu} B^{1-\nu}\right)\right)^{m} \\
& +r_{0}^{n m}\left(\left(\operatorname{det}(A)^{\frac{1}{n}}+\operatorname{det}(B)^{\frac{1}{n}}\right)^{n m}-2^{n m}(\operatorname{det}(A B))^{\frac{m}{2}}\right) \\
& +r_{n m}\left[\left(\operatorname{det}(B)^{\frac{1}{n}} \frac{\operatorname{det}(B)^{m}-\operatorname{det}(A)^{m}}{\operatorname{det}(B)^{\frac{1}{n}}-\operatorname{det}(A)^{\frac{1}{n}}}+\operatorname{det}(A B)^{\frac{m}{2}}-(n m+1)\left(\operatorname{det}\left(A^{m} B^{m+\frac{2}{n}}\right)\right)^{\frac{n m}{2(n m+1)}}\right.\right. \\
& \times \chi_{\left(0, \frac{1}{2}\right]}(\nu) \\
& \left.+\left(\operatorname{det}(A)^{\frac{1}{n}} \frac{\operatorname{det}(B)^{m}-\operatorname{det}(A)^{m}}{\operatorname{det}(B)^{\frac{1}{n}}-\operatorname{det}(A)^{\frac{1}{n}}}+\operatorname{det}(A B)^{\frac{m}{2}}-(n m+1)\left(\operatorname{det}\left(A^{m+\frac{2}{n}} B^{m}\right)\right)^{\frac{n m}{2(n m+1)}}\right)\right] \\
& \times \chi_{\left(\frac{1}{2}, 1\right]}(\nu) .
\end{aligned}
$$

The second result of this section concerns the traces of positive definite matrices which can be reads as follows:

Theorem 3.2. Let $A, B \in \mathbf{M}_{n}(\mathbf{C})$ be positive definite matrices and $0 \leq$ $\nu \leq 1$. Then for $m=1,2,3, \ldots$, we have

$$
\begin{aligned}
& \left(\operatorname{tr}\left(\left|A^{\nu} B^{1-\nu}\right|\right)^{m}+r_{0}^{m}\left((\operatorname{tr}(A)+\operatorname{tr}(B))^{m}-2^{m}(\operatorname{tr}(A) \operatorname{tr}(B))^{\frac{m}{2}}\right)\right. \\
& +r_{m}\left[\left(\operatorname{tr}(A) \frac{(\operatorname{tr} B)^{m}-(\operatorname{tr} A)^{m}}{\operatorname{tr} B-\operatorname{tr} A}+(\operatorname{tr}(A) \operatorname{tr}(B))^{\frac{m}{2}}-(m+1)\left((\operatorname{tr} A)^{m}(\operatorname{tr} B)^{m+2}\right)^{\frac{m}{2(m+1)}}\right)\right. \\
& \times \chi_{\left(0, \frac{1}{2}\right]}(\nu) \\
& +\left(\operatorname{tr}(B) \frac{(\operatorname{tr} B)^{m}-(\operatorname{tr} A)^{m}}{\operatorname{tr} B-\operatorname{tr} A}+(\operatorname{tr}(A) \operatorname{tr}(B))^{\frac{m}{2}}-(m+1)\left((\operatorname{tr} A)^{m+2}(\operatorname{tr} B)^{m}\right)^{\frac{m}{2(m+1)}}\right) \\
& \left.\times \chi_{\left(\frac{1}{2}, 1\right]}(\nu)\right] \\
& \leq[\operatorname{tr}(\nu A+(1-\nu) B)]^{m},
\end{aligned}
$$


where $r_{0}=\min \{\nu, 1-\nu\}$, and $r_{m}=\min \left\{2^{m} r_{0}^{m} ;\left(\begin{array}{c}m \\ k\end{array}\right)\left(r_{0}^{k}\left(1-r_{0}\right)^{m-k}-\right.\right.$ $\left.\left.r_{0}^{m}\right), 0 \leq k \leq m-1\right\}$.

Proof. We have

$$
\begin{gathered}
\left(\operatorname{tr}\left(\left|A^{\nu} B^{1-\nu}\right|\right)^{m}+r_{0}^{m}\left((\operatorname{tr}(A)+\operatorname{tr}(B))^{m}-2^{m}(\operatorname{tr}(A) \operatorname{tr}(B))^{\frac{m}{2}}\right)\right. \\
+r_{m}\left[\left(\operatorname{tr}(A) \frac{(\operatorname{tr} B)^{m}-(\operatorname{tr} A)^{m}}{\operatorname{tr} B-\operatorname{tr} A}+(\operatorname{tr}(A) \operatorname{tr}(B))^{\frac{m}{2}}-(m+1)\left((\operatorname{tr} A)^{m}(\operatorname{tr} B)^{m+2}\right)^{\frac{m}{2(m+1)}}\right)\right. \\
\times \chi_{\left(0, \frac{1}{2}\right]}(\nu) \\
+\left(\operatorname{tr}(B) \frac{(\operatorname{tr} B)^{m}-(\operatorname{tr} A)^{m}}{\operatorname{tr} B-\operatorname{tr} A}+(\operatorname{tr}(A) \operatorname{tr}(B))^{\frac{m}{2}}-(m+1)\left((\operatorname{tr} A)^{m+2}(\operatorname{tr} B)^{m}\right)^{\frac{m}{2(m+1)}}\right) \\
\left.\quad \times \chi_{\left(\frac{1}{2}, 1\right]}(\nu)\right] \\
\leq\left[(\operatorname{tr} A)^{\nu}(\operatorname{tr} B)^{1-\nu}\right]^{m}+r_{0}^{m}\left((\operatorname{tr}(A)+\operatorname{tr}(B))^{m}-2^{m}(\operatorname{tr}(A) \operatorname{tr}(B))^{\frac{m}{2}}\right) \\
+r_{m}\left[\left(\operatorname{tr}(A) \frac{(\operatorname{tr} B)^{m}-(\operatorname{tr} A)^{m}}{\operatorname{tr} B-\operatorname{tr} A}+(\operatorname{tr}(A) \operatorname{tr}(B))^{\frac{m}{2}}-(m+1)\left((\operatorname{tr} A)^{m}(\operatorname{tr} B)^{m+2}\right)^{\frac{m}{2(m+1)}}\right)\right. \\
\times \chi_{\left(0, \frac{1}{2}\right]}(\nu) \\
+\left(\operatorname{tr}(B) \frac{(\operatorname{tr} B)^{m}-(\operatorname{tr} A)^{m}}{\operatorname{tr} B-\operatorname{tr} A}+(\operatorname{tr}(A) \operatorname{tr}(B))^{\frac{m}{2}}-(m+1)\left(\operatorname{tr}(A)^{m+2} \operatorname{tr}(B)^{m}\right)^{\frac{m}{2(m+1)}}\right) \\
\left.\times \chi_{\left(\frac{1}{2}, 1\right]}(\nu)\right] \\
\quad(\operatorname{by~inequality~}(3.4)) \\
\leq \quad\left[\operatorname{tr}(\nu A+(1-\nu) B]^{m}(\text { by Theorem } 2.1) .\right.
\end{gathered}
$$

The third result of this section concerns the norms of positive semidefinite matrices which can be reads as follows:

Theorem 3.3. Let $A, X, B \in \mathbf{M}_{n}(\mathbf{C})$ be positive semi-definite matrices and $0 \leq \nu \leq 1$. Then for $m=1,2,3, \ldots$,

$$
\| A^{\nu} X B^{1-\nu}||^{m}+r_{0}^{m}\left(\left(|||A X|\|+|\| X B|||)^{m}-2^{m}(|||A X||||| X B|| \mid)^{\frac{m}{2}}\right)\right.
$$




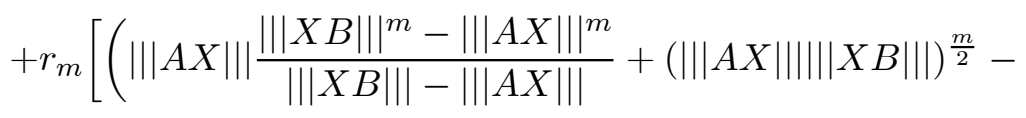

$$
\begin{aligned}
& (m+1)\left(\left\||| A X\left|\left\|\left.\right|^{m}|\|X B\||^{m+2}\right)^{\frac{m}{2(m+1)}}\right) \times \chi_{\left(0, \frac{1}{2}\right]}(\nu)\right.\right.
\end{aligned}
$$

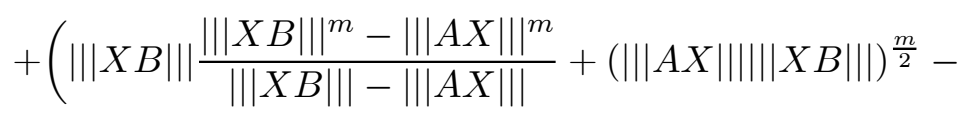

$$
\begin{aligned}
& \left.(m+1)\left(|||A X|||^{m+2}|| X B \mid \|^{m}\right)^{\frac{m}{2(m+1)}}\right) \times \chi_{\left(\frac{1}{2}, 1\right]}(\nu) \\
& \leq\left[\nu \left|\left\|A X \left|\left\|+(1-\nu)\left|\|X B|\||]^{m}\right.\right.\right.\right.\right.\right.
\end{aligned}
$$

where $r_{0}=\min \{\nu, 1-\nu\}$, and $r_{m}=\min \left\{2^{m} r_{0}^{m} ;\left(\begin{array}{c}m \\ k\end{array}\right)\left(r_{0}^{k}\left(1-r_{0}\right)^{m-k}-\right.\right.$ $\left.\left.r_{0}^{m}\right), 0 \leq k \leq m-1\right\}$.

\section{Proof. We have}

$$
\begin{aligned}
& \|\left. A^{\nu} X B^{1-\nu}||\right|^{m}+r_{0}^{m}\left(\left((|||A X|||+|||X B|||)^{m}-2^{m}(|||A X||||| X B|| \mid)^{\frac{m}{2}}\right)\right. \\
& +r_{m}\left[\left(\||| A X|| \mid \frac{\left\||X B|||^{m}-\right\||| A X||^{m}}{\||X B|||-|||A X|||}+(|||A X||||||X B|||)^{\frac{m}{2}}-\right.\right. \\
& (m+1)\left(\left|\left\|\left.A X||\right|^{m}|||X B|\right\|^{m+2}\right)^{\frac{m}{2(m+1)}}\right) \times \chi_{\left(0, \frac{1}{2}\right]}(\nu) \\
& +\left(\||X B||| \frac{\left\|\left.X B\left|\left\|^{m}-\right\|\right||A X|\right|^{m}\right.}{\|X B|\|-||| A X|| \mid}+(\||| A X|||||| X B|| \mid)^{\frac{m}{2}}-\right. \\
& (m+1)\left(\left|\left\|\left.A X||\right|^{m+2} \mid\right\| X B \|^{m}\right)^{\frac{m}{2(m+1)}}\right) \times \chi_{\left(\frac{1}{2}, 1\right]}(\nu) \\
& \leq\left[\left.\left.\||A X|\|\right|^{\nu}\||X B|\|\right|^{1-\nu}\right]^{m} \\
& \left\|A^{\nu} X B^{1-\nu} \mid\right\|^{m}+r_{0}^{m}\left((\||| A X|\|+\|||X B|\|)^{m}-2^{m}(|||A X||||| X B|| \mid)^{\frac{m}{2}}\right) \\
& +r_{m}\left[\left(\||| A X|| \mid \frac{\left.|| X B\left|\left\|^{m}-\right\| A X\right|\right|^{m}}{\|X B|\|-\| A X|||}+(\||| A X|||||| X B|| \mid)^{\frac{m}{2}}-\right.\right. \\
& (m+1)\left(\left|\left\|A X||^{m}|||X B|\right\|^{m+2}\right)^{\frac{m}{2(m+1)}}\right) \times \chi_{\left(0, \frac{1}{2}\right]}(\nu)
\end{aligned}
$$




$$
\begin{aligned}
& +\left(\||| X B|| \mid \frac{\|\left. X B||\right|^{m}-|||A X|||^{m}}{\|X B|\|-||| A X|| \mid}+(\||| A X|||||| X B|| \mid)^{\frac{m}{2}}-\right. \\
& (m+1)\left(\|\| A X\left|\left\|^{m+2} \mid\right\| X B \|^{m}\right)^{\frac{m}{2(m+1)}}\right) \times \chi_{\left(\frac{1}{2}, 1\right]}(\nu)
\end{aligned}
$$

( by inequality (3.3))

$$
\leq\left[\nu \left|\left\|A X \left|\|+(1-\nu)|\|X B\|| \mid]^{m}\right.\right.\right.\right. \text { (by Theorem 2.1). }
$$

\section{Acknowledgements.}

The authors would like to express their deep thanks to the anonymous referees for their helpful comments and suggestions on the initial version of the manuscript which lead to the improvement of this paper.

\section{References}

[1] Y. Al- Manasrah and F. Kittaneh, "A generalization of two refined Young inequalities”, Positivity, vol. 19, pp. 757-768, 2015.

[2] Y. Al- Manasrah and F. Kittaneh, "Further Generalization, Refinements, and Reverses of the Young and Heinz Inequalities", Results in Mathematics, vol. 19, pp. 757-768, 2016.

[3] M. Akkouchi and M. A. Ighachane, "A new proof of a refined Young inequality", Bulletin of the International Mathematical Virtual Institute, vol. 10, no. 3, pp. 425-428, 2020.

[4] T. Ando, "Matrix Young Inequalities," in Operator Theory in Function Spaces and Banach Lattices, C. B. Huijsmans, M. A. Kaashoek, W. A. J. Luxemburg, and B. Pagter, Eds. Basel: Birkhäuser, 1995, pp. 33-38.

[5] S. Furuichi, "On refined Young inequalities and reverse inequalities", Journal of Mathematical Inequalities, vol. 5, pp. 21-30, 2011.

[6] R. A. Horn and C. R. Johnson, Matrix analysis. New York: Cambridge University. Press, 1985.

[7] O. Hirzallah, and F. Kittaneh, "Matrix Young inequalities for the Hilbert-Schmidt norm", Linear Algebra and its Applications, vol. 308, pp. 77-84, 2000.

[8] T. Kato, "Notes on some inequalities for linear operators", Mathematische Annalen, vol. 125, pp. 208-212, 1952. 
[9] M. A. Ighachane, M. Akkouchi, and El Hassan Benabdi, "A new generalized refinement of the weighted arithmetic-geometric mean inequality", Mathematical Inequalities and Applications, vol. 23, no. 3, pp. 1079-1085, 2020.

[10] M. A. Ighachane and M. Akkouchi, "A new generalization of two refined Young inequality", Moroccan Journal of Pure and Applied Analysis, vol. 6, no. 2, pp. 155-167, 2020.

[11] F. Kittaneh, "Norm inequalities for fractionl powers of positive operators”, Letters in Mathematical Physics, vol. 27, pp. 279-285, 1993.

[12] F. Kittaneh and Y. Al- Manasrah, "Improved Young and Heinz inequalities for matrices", Journal of Mathematical Analysis and Applications, vol. 361, pp. 292-269, 2010.

[13] J. Zhao and J. Wu, "Operator inequalities involving improved Young and its reverse inequalities", Journal of Mathematical Analysis and Applications, vol. 421, pp. 1779-1789, 2015.

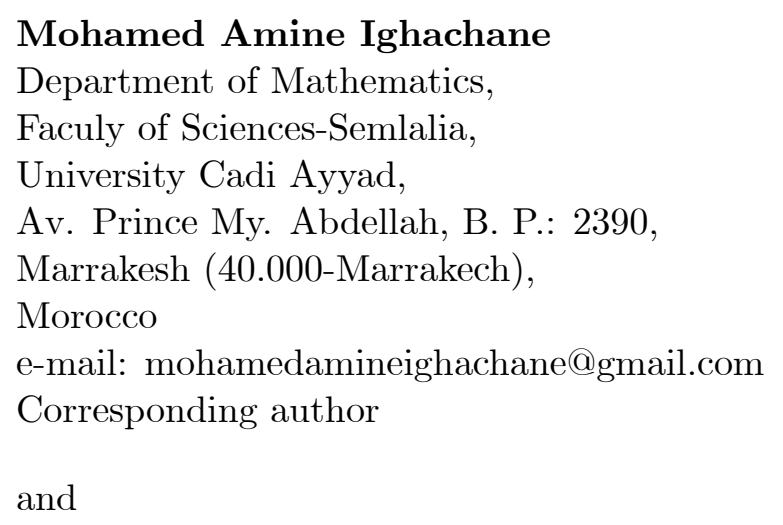

Mohamed Akkouchi

Department of Mathematics, Faculy of Sciences-Semlalia, University Cadi Ayyad, Av. Prince My. Abdellah, B. P.: 2390, Marrakesh (40.000-Marrakech), Morocco e-mail: akkm555@yahoo.fr 